\title{
Retinal hazards during laser irradiation of the iris
}

\author{
G. K. WATTS
}

Department of Experimental Ophthalmology, Institute of Ophthalmology, University of London

Transpupillary pulsed laser radiation has been extensively studied to determine both the level at which it becomes a hazard to the retina (Ham, Williams, Mueller, Guerry, Clarkc, 응 and Geeraets, I966), and its effects on that tissue when such levels are exceeded (Marshall of and Mellerio, r $967 \mathrm{a}, \mathrm{b}, \mathrm{rg68})$. In spite of the development of various codes of practice to protect users, several accidents have been reported (Rathkey, I965; Zweng, 1967; 8 Curtin and Boyden, 1968). More recently an attempt has been made to evaluate trans- $\frac{\rho}{2}$ scleral hazards (Smith and Stein, 1968, 1969), and safe levels have now been defined for both ruby and neodymium laser beams entering the eye through this tissue.

No investigative work has so far been carried out on the possible transmission of laser beams impinging on the iris. Such an event might take place under two circumstances; first, with the eye open the iris may contribute up to half the exposed area of the globe and thus form a significant target for laser beams accidentally discharged towards the eye. Secondly, it may be necessary to fire laser beams directly at the iris for clinical reasons; peripheral iridotomy by laser has been discussed in the literature (Flocks and Zweng, I964; Hallman, Perkins, Watts, and Wheeler, 1968), and retinal hazards commented upon (Snyder, 1967), but no practical measurements of such hazards have been made.

The aim of this work is to study the spectral characteristics of the iris to non-coherent light and (to a limited extent) laser light, and predict the possible hazards of trans-iridial laser radiation.

\section{Experimental methods}

\section{A. NON-COHERENT LIGHT}

The first problem in attempts to measure the spectral characteristics of the iris was the mounting of the specimens. The tissue stretches easily and thus required not only a firm base but careful control of its thickness. The thickness of the iris in vivo varies with the size of the pupil, and the following method was designed to give a preparation which could be handled easily and ensure that each iris was examined under similar conditions. Although the absolute values obtained with this method may not correspond to the iris in its normal state, comparative values for transmission and reflectance at different wavelengths and with different coloured irides should still be valid.

The iris samples were prepared from freshly enucleated rabbit eyes; the dissection procedure was simple but, in view of the relatively fragile nature of the unfixed iris, extreme care was required. The posterior segment of the globe was cut away and the anterior segment was held face downwards in a dish while capsule forceps were slipped beneath the lens to lift it away from the underlying pigment epithelial layers of the iris. The iris was removed by seizing its periphery with a pair of toothed forceps and gently tugging. Provided that reasonable care was taken, the whole tissue could then be removed intact. A single radial cut from pupil to periphery enabled the iris to be laid flat on a microscope slide in a pool of saline and smoothed out. A second slide was then placed over the preparation and the tissue compressed until it had only the thickness of the single cover 
slip placed between the slides at either end. Excess saline was wiped away with filter paper and the ends of the slides were bound together with adhesive tape. In this way consistent preparations could be made.

Each preparation was mounted over the window of the cell-holder of a Unicam S.P. 500 spectrophotometer, and transmission values were measured in steps of $500 \AA$. Before each measurement, the photocell dark-current was zeroed and a control blank of two plane glass microscope slides was placed in the beam path; the aperture of the photocell was opened and the machine re-zeroed, thus correcting for absorption due to the glass. The same tungsten light source was used for the whole wavelength range, but two photo cells were necessary: a red-sensitive one between i i, ooo and $6,250 \AA$ and a blue-sensitive one over the rest of the range.

In addition to the preparation of iris, a model experiment was carried out on a melanin suspension. Two slides were prepared, one coated with 5 per cent. gelatin and the other with a suspension of $15 \mathrm{mg}$. $/ \mathrm{ml}$. powdered melanin in 5 per cent. gelatin. These slides were scanned over the same wavelength range in the spectrophotometer, the gelatin-only slide serving as a control.

For determination of the reflectance of the irides, the same method of preparation was adopted and the specimens scanned in a Beckman recording spectroreflectometer. This intsrument works by comparing the reflectivity of the test surface with that of a standard reflector, the differences between the two being recorded by the movement of a pen over a chart calibrated according to wavelength. Eight albino, eight brown, and three blue irides, each with the minimum area of Io $\times 6 \mathrm{~mm}$. required by the apparatus, were prepared and mounted as described. Each sample was placed behind one window of the measuring sphere of the apparatus and a standard white tile behind the other, the pair then being scanned over the wavelength range 5,000 $\AA$ to I I,000 $\AA$. The reflectance values at various wavelengths were read at $500 \AA$ intervals from each of the recordings, and the mean at each wavelength was calculaced. At this stage all readings represented percentage reflections relative to the white standard tile which was itself not 100 per cent. reflecting; to arrive at the final percentages, a correction had to be made to each mean reading from a graph of the known absolute reflection characteristics of the tile as supplied by its manufacturers. The final percentage reflections were plotted as a function of the incident wavelength.

\section{B. LASER LIGHT}

The experiments with laser light were restricted to the passive ruby laser and fell into two categories:

(1) The first series of experiments was designed to measure the absolute transmission of a laser beam through the iris by direct calorimetric recording. The iris was dissected out of each specimen as previously described and placed, pigment epithelium downwards, on a cover slip by which it was transferred to a specially constructed glass cell. A blunt seeker was used to manoeuvre the iris from the cover slip onto the inner wall of the rear face of the cell where surface tension sufficed to hold it in place. The cell was then filled with a silicone fluid (Midland Silicones, 200/l cSt) and the rear surface was optically masked with black paper, leaving only a hole the size and shape of the tissue. The cell was mounted vertically in front of, and closely opposed to, the measuring cone of a calorimeter (Laser Associates) so that all light passing through the iris would enter the measuring device no matter how much it deviated. The laser, mounted on a Gambs photo slit lamp (Hallman and others, 1968) was aligned in front of the cell so that its beam was focused at the plane of the iris. The cell was movable by means of a vertical and horizontal racking device, so that any part of the iris could be lasered and its transmission recorded. Between eight and fifteen lesions were made on each of the irides used, and the mean transmission was calculated from the recordings; at the end of the experiment the incident energy was measured by firing the laser six times into the calorimeter through cell and fluid alone, the iris having been removed. A beam-splitting device enabling individual shots to be monitored would have been preferable but was not available, and so the experiments were based on the assumption of stability in the laser output. To minimize variation in output, the firings were separated by a fixed time interval of $5 \mathrm{~min}$. The percentage transmission of the irides was calculated from the mean transmission values and mean output of the laser. 
A slight variation of this method was used in the case of an albino iris; instead of using the same incident energy at each firing, the transmission was measured at the full range of switched energy steps available from the laser. Incident and transmitted energies at each level were plotted against one another, the result being a straight-line graph, the slope of which gave another indication of percentage transmission.

(2) The geometry of the transmitted beam was investigated to discover whether it remained a discrete, though less energetic, beam or was diffused by its passage through the tissue. The principle of the experiment was similar to the previous one except that instead of the calorimeter, an unexposed Ilford R. Io photographic plate was placed directly behind the cell. In one experiment the os developed plate was replaced behind the cell in an identical position to that which it had occupied $\vec{O}$ before exposure, the iris was removed, and the laser fired again. The plate, blackened by development, presented an absorbing filter to the beam which caused a burn on the gelatin film indicating where the original beam would have impinged in the absence of the iris. Other exposed and develop- $\frac{O}{O}$. ed plates were then searched in corresponding areas for evidence of an undiffused beam. To relate $\dot{v}$ the experiment more closely to the condition in vivo, it was repeated with a whole anterior segmentiris, cornea, and lens-held together by a $3 \mathrm{~mm}$. rim of sclera. The preparation was suspended $\delta$ in a similar but deeper glass cell by cotton sutures through the scleral rim, and subjected to the same $O$ treatment.

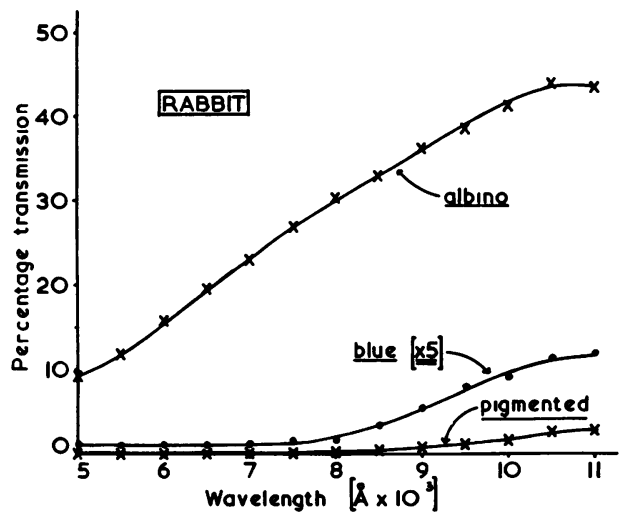

FIG. I Spectrophotometer transmission readings of rabbit irides compressed to a uniform thickness between two microscope slides. The values for the blue iris are shown multiplied by 5 to separate them from those of the brown iris

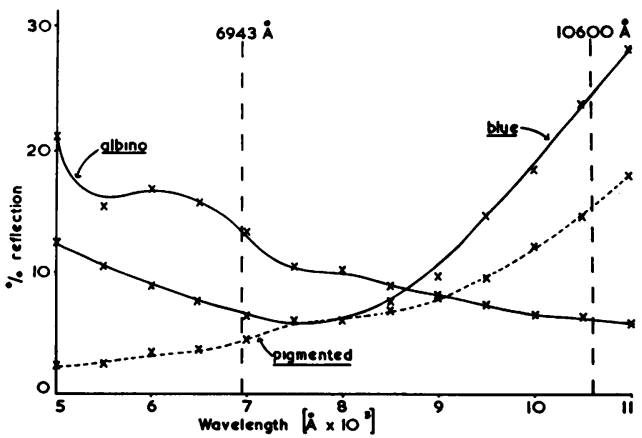

FIG. 3 Reflectivity of blue, brown (pigmented), and albino rabbit irides as measured in a spectroreflectometer

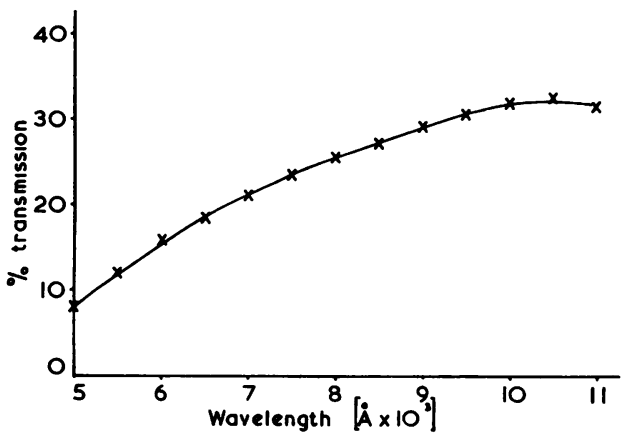

FIG. 2 Transmission of a suspension of melanin granules in gelatin

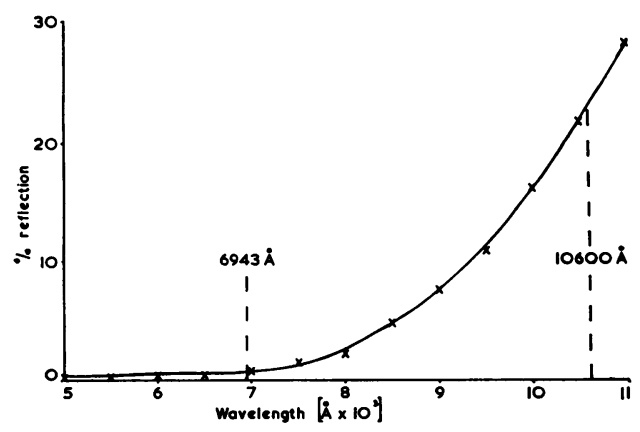

FIG. 4 Reflectivity of the pigment epithelium of $a$ pigmented rabbit iris 


\section{Results}

The measurements of non-coherent light transmission and reflection are presented graphically (Figs I to 4), and the transmission of the laser numerically (Table). The pigmented rabbit iris irradiated with $200 \mathrm{~mJ}$ of ruby laser light yielded a transmission value so far

Table Mean transmission in a series of experiments with irides of different colours

\begin{tabular}{|c|c|c|c|}
\hline Iris type & No. of recordings & $\begin{array}{l}\text { Mean transmission } \\
\text { (per cent.) }\end{array}$ & $\begin{array}{l}\text { Standard } \\
\text { deviation }\end{array}$ \\
\hline Albino & 75 lesions in five eyes & $30 \cdot 1$ & $1 \cdot 8$ \\
\hline Blue & 43 lesions in four eyes & $4 \cdot 2$ & $1 \cdot 7$ \\
\hline Brown & 60 lesions in five eyes & $<1$ & 一 \\
\hline
\end{tabular}

below I per cent. that it was not considered either valid or useful to measure it precisely. The value of 30 per cent. for the albino iris is in good agreement with the value of 29 per cent. calculated from the graph of transmission at various incident energies (Fig. 5).

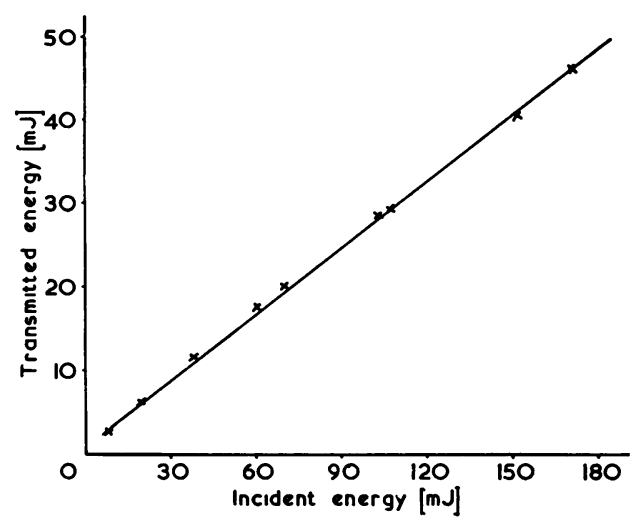

FIG. 5 Transmission at various energies of an albino rabbit iris

The photographic recordings revealed no indication of a discrete spot on the plates behind either the pigmented or albino rabbit irides (Figs $6 a$ and $b$, overleaf). Although the plates were located directly behind the cell, and therefore only a few millimetres from the back of the lesion, albino irides caused the emulsion to be fogged over a roughly circular area $3 \mathrm{~cm}$. in diameter. The results, both from isolated irides and whole anterior segments, were similar (Figs $6 c$ and $d$, overleaf).

\section{Discussion}

Several papers have been published concerning light transmission through the ocular media (Wiesinger, Schmidt, Williams, Tiller, Ruffin, Guerry, and Ham, 1956), the retina and choroid (Geeraets, Everett, and Guerry, 1962), and even the sclera (Smith and Stein, 1968). The iris, however, has escaped attention. As mentioned earlier, its contractile nature leads to variations in thickness which would possibly alter its transmission characteristics. The figures quoted here for laser transmissions must be regarded only as indications of the levels to be expected. 

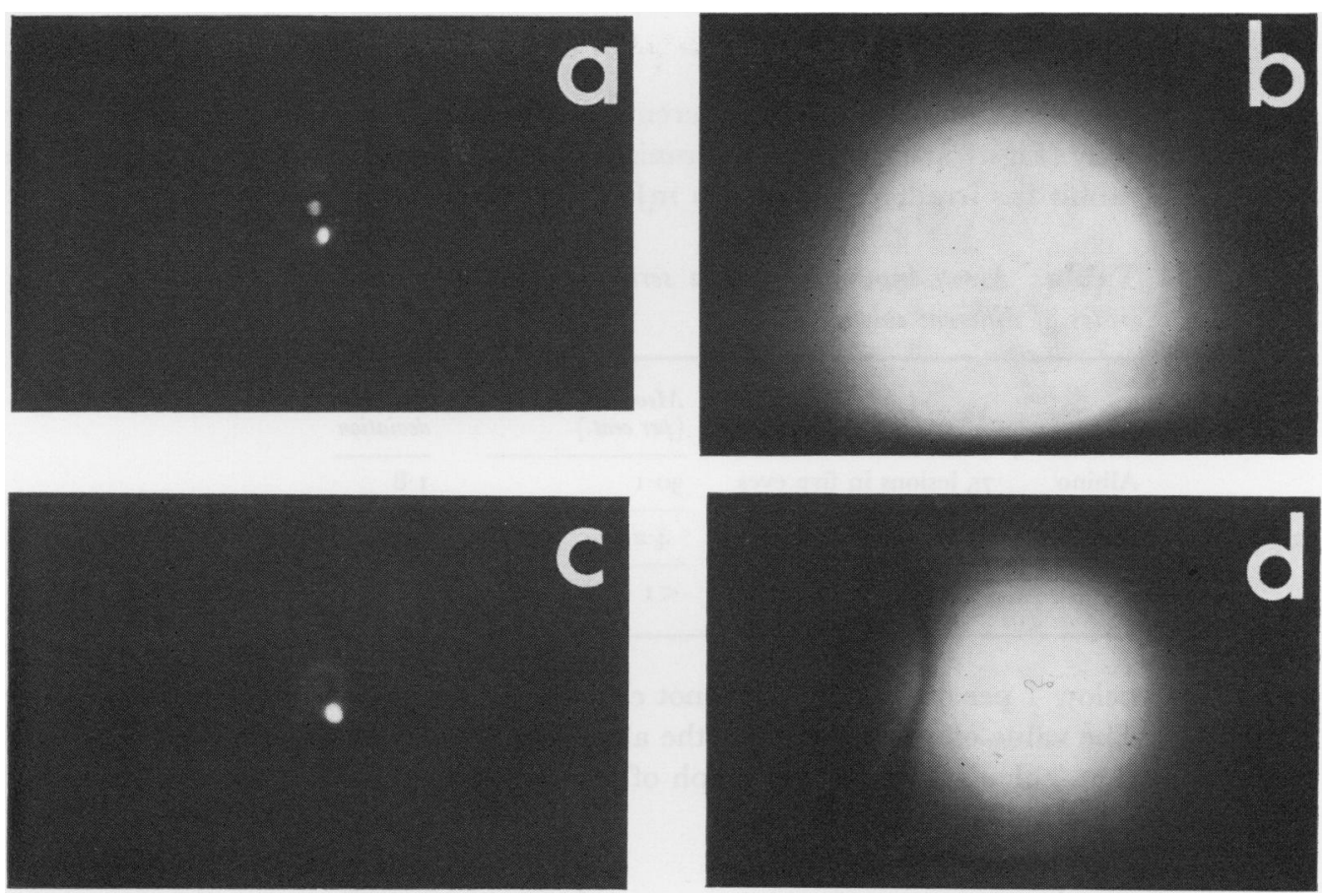

FIG. 6 Photographic recording of the transmission of a passive ruby laser beam through the albino rabbit iris

(a) The unimpeded beam before interposition of the iris into the cell between laser and photographic plate The beam is the lower and more intense of the two white spots; the upper is a reflection artefact from the walls of the glass cell

(b) The beam after trans-iridial passage

(c) As (a), but using a deeper cell

(d) The beam after passage across a whole anterior segment instead of an iris alone

A few workers have examined uveal pigment either isolated (Pakkenberg, 1966) or in sections of the iris (Jensen, 1969), but no studies appear to have been performed on the properties of the whole tissue. Figs $\mathrm{I}$ and 2 indicate that increase in transmission with wavelength is a general property of both the tissue and its pigment over the range examined; this is comparable with the findings of Pakkenberg ( 1966) on individual pigment cells over the same region of the visible spectrum. It might be argued that this apparent increase in transmission is merely a by-product of a decreased reflection (which would 9 make more incident energy available) at longer wavelengths. Fig. 3 reveals that this is not the case, at least for the blue and brown irides which actually reflect more at the longer wavelengths. The albino iris does not show this tendency, which partly accounts for the steep rise in transmission in Fig. I compared with the curves for the pigmented irides. The general slope of the curves, including the suggestion of a peak around IoI I , ooo $\AA$, resembles Smith and Stein's results for the combined sclera, choroid, and retina.

The reflection curves of the iris are slightly more complicated than its transmission curves, and pigment-free tissue exhibited properties different from those of its isolated pigment. Inspection of Fig. 4 shows that reflection from the pigment alone increases at longer wavelengths while that from albino tissue (Fig. 3) decreases. The U-shaped curve for the blue iris (Fig. 3) may thus be seen as a combination of high reflection from the tissue stroma at short wavelengths and from the pigment epithelium at longer wave- 
lengths. No explanation can be advanced for the slight hump in the albino curve at 6, ooo $\AA$, but a similar peak was found by Smith and Stein (1968) in their studies on reflectance of the rabbit sclera, another pigment-free tissue.

The curves in Figs $I$ and 3 suggest that the iris will react differently to the two most commonly used pulsed lasers (ruby at $6,943 \AA$ and neodymium at ro,6oo $\AA$ ). The pigmented rabbit iris reflects a greater proportion of neodymium than of ruby laser light; furthermore, of the reduced amount of neodymium laser energy actually penetrating the surface of the iris, more is transmitted through it. The total absorption is thus lower and the neodymium laser represents a smaller hazard to the iris tissue than the ruby laser. This slight disparity of incident energy (further enhanced by the extra absorption of near infra-red light in the ocular media) is apparent in the lesions caused by a neodymium laser in vivo (unpublished data). As regards damage to the fundus, the neodymium laser with its higher transmission represents the greater danger, and in clinical work such as iridotomy where the object would be to confine damage to the iris, it is quite clearly contraindicated by comparison with the ruby laser. Optimum clinical results should (by reference to Figs I and 3) be achieved using a pulsed laser with a shorter wavelength than the ruby laser. While having a high transmission in the ocular media, reflection from the surface of the iris would be low and absorption within its depth would be high. For example, the frequency-doubled neodymium laser operating at 5,300 $\AA$ (yellow-green) should give better results, but the difference might well prove to be marginal.

Densitometric studies of unstained sections of the brown rabbit iris (Hallman and others, I968) indicate that the bulk of the tissue pigment is in the pigment epithelium, the remainder being concentrated in the anterior layers of the stroma. The blue iris is entirely devoid of stromal pigment, but has a normal epithelium. The similarity of the laser transmission results for the blue and brown rabbit irides is thus not surprising; compared with the pigment in the epithelium, the stromal pigment of the rabbit represents a minimal barrier.

Accepting that a little light does pass the pigment epithelium, the photographic recordings of the transmitted beam would seem to indicate that it is very unlikely to cause retinal damage. The iris acts not only as an optical filter (Fig. 5) but the photographs (Fig. 6) indicate that it also acts as a very efficient diffuser of the laser beam.

In an attempt to relate this work directly to the human iris, several cadaver eyes were obtained from the Moorfields Hospital Eye Bank, and subjected to laser transmission studies identical to those performed on the rabbit. However, despite refrigeration for the 2 to 3 day period between enucleation and use, the pigment epithelium tends to be dislodged more easily after storage (Kaczurowski, 1965), and it is more susceptible to damage during dissection, an important point if it is borne in mind that this single cell layer plays a major role in light absorption. The transmission values of the human iris showed considerably more scatter than those of the rabbit, and the mean values were 5.5 per cent. (S.D. $2 \cdot 8$ per cent.) for a brown iris ( 30 lesions on 3 eyes), and 14 per cent. (S.D. 6.3 per cent.) for a blue iris (29 lesions on 3 eyes). It is difficult to calculate the energy density incident upon the retina due to transiridial passage, but it must be well below the damage threshold. This is generally considered to be $0.7 \mathrm{~J} / \mathrm{cm} .{ }^{2}$ (Ham and others, I966) so that, even if 20 per cent. of a $200 \mathrm{~mJ}$ clinical exposure was transmitted, it would represent only $0.04 \mathrm{~J}$ over the whole of the fundus. The only danger would arise if a laser was discharged at an area of the iris which had already undergone atrophy (Snyder, 1967); retinal damage might then be sustained. However, prior transillumination of the iris should be sufficient to reveal any such areas. 
Assuming that the light transmitted through the iris is dispersed evenly over the posterior half of the globe, calculation shows that the incident beam may have an energy density of several hundred $\mathrm{J} / \mathrm{cm} .^{2}$ before the retinal threshold is reached. However, there is no guarantee that the diffusing properties of the iris will remain the same at high energies; that proportion of the laser beam absorbed as a result of the optical filtering by tissue causes it to undergo partial destruction. In a beam approximately $\mathrm{I} \cdot 2 \mathrm{~mm}$. diameter, destruction starts at an energy of about $15 \mathrm{~m} \mathrm{~J}$, i.e. an energy density of $\mathrm{I} \cdot \mathrm{I} J / \mathrm{cm} .{ }^{2}$, and at $16 \mathrm{~J} / \mathrm{cm} .^{2}$, the pigment epithelium is completely shattered. It may then be assumed that, above $16 \mathrm{~J} / \mathrm{cm} .{ }^{2}$, the percentage of laser light transmitted through a brown iris will increase; ${ }^{\text {c }}$ it is also possible that the damage to the iris will not only permit the passage of more light, but that this light may be in a more geometrically concentrated beam and will therefore constitute a greater hazard.

Nothing has yet been said of the structure closest to the iris, the lens. Since its epithelium is closely apposed to the pigment epithelium of the iris, cataractogenetic heating or of the lens surface might be suspected. Indeed, this is the subject of a recent paper by Leibowitz and Luzzio (1970), who produced numerous cataracts with a ruby laser at an energy density of $29.5 \mathrm{~J} / \mathrm{cm} .^{2}$. However, at the level of about $16 \mathrm{~J} / \mathrm{cm} .^{2}$ used in this work, no sign of cataract was seen in rabbits either killed immediately or kept for periods of up to 18 months. On the basis of Leibowitz and Luzzio's data, a few cataracts might have been expected in this work. The explanation probably lies in the differing experimental conditions; Leibowitz and Luzzio lasered the whole anterior segment of the eye $\vec{\oplus}$ while lesions in the present work were localized and only a little over I $\mathrm{mm}$. in diameter. Thus, while the energy densities were not so far removed, the absolute energies used differed by perhaps two orders of magnitude. In a small closed-box system like the eye, this would tend to render the experiments incomparable. However, while lens damage constitutes a potential hazard, it is not strictly within the province of this paper and will be discussed no further.

\section{Conclusions}

(r) The neodymium laser applied to the iris constitutes a greater retinal hazard than the ruby laser.

(2) The stored human irides used in this work exhibited a higher transmission than did those of the rabbit eyes.

(3) The iris acts as an optical diffuser as well as an attenuator.

(4) Transmitted ruby laser light from a clinical exposure to the iris of up to $16 \mathrm{~J} / \mathrm{cm} .{ }^{2}$ 음 is unlikely to induce retinal damage.

(5) Above $16 \mathrm{~J} / \mathrm{cm}^{2}$, the integrity of the pigment epithelium is lost and the diffusing properties of the iris may well decline, thus disproportionately increasing the dangers from transmitted energy.

\section{Summary}

Rabbit irides (albino, blue, and brown) were subjected to spectrophotometry and spectroreflectometry to assess their transmission and reflection properties to light between 5,000 ? and $\mathrm{I} I, 000 \AA$. The geometry and energy of a ruby laser beam transmitted through the iris were assessed by placing a photographic plate and calorimeter respectively behind a test specimen during irradiation. Several irides were similarly investigated. 
From this data, the possible hazards to the retina from clinical or accidental exposure of the iris were evaluated.

I should like to thank Prof. E. S. Perkins for advice and encouragement and Mr. M. S. Bass for his skilled technical assistance.

Dr. K. Rassum of Imperial College, London, kindly provided facilities for the reflection measurements. This work was supported by a grant from the Ministry of Defence.

\section{References}

Gurtin, T. L., and Boyden, D. G. (1968) Amer. F. Ophthal., 65, i 88 Flocks, м., and zWeng, H. c. (1964) Arch. Ophthal. (Chicago), 72, 604 geeraets, w. J., EVERETt, w. G., and GUerry, D. (ig62) Amer. F. Ophthal., 54, 393 hallman, v. L., PERkins, E. S., WAtts, G. K., and wheeler, c. B. (1968) Exp. Eye Res., 7, 48I ham, W. T., Williams, R. C., MUEller, H. A., GUerRy, D., Glarke, A. M., and Geeraets, W. J. (ig66)

Trans. N.Y. Acad. Sci., ser. II, 28, 5 I 7 JENSEN, s. (1969) Acta ophthal. (Kbh.), 47,625

KACZURowski, м. I. (1965) Amer. J. Ophthal., 59, 299

Leibowitz, H. м., and Luzzio, A. J. (1970) Arch. Ophthal. (Chicago), 83, 608

MARSHALl, J., and MELLERIo, J. (1967a) Exp. Eye Res., 6, 4

$\longrightarrow$ (1967b) Ibid., 6, 303

PAKKeNBERG, H. (1966) Brain Res., 2, I 73

RAThKey, A. S. (1965) Arch. Ophthal. (Chicago), 14, 346

SMith, R. s., and STEIn, м. N. (I968) Amer. F. Ophthal., 66, 2 I

$\longrightarrow,-(1969) \quad$ Ibid., 67, 100

SNYDER, w. B. (1967) Arch. Ophthal. (Chicago), 77, 93

WIESINGER, H., SCHMIDT, F. H., WILliAMS, R. G., TILlER, C. O., RUFFIN, R. S., GUERRY, D., and HAM, W. T.

(1956) Amer. F. Ophthal., 42, 907

zWENG, H. c. (1967) Arch. Ophthal. (Chicago), 78, 596 